\title{
A Hybrid Carbon Price Forecasting Model with External and Internal Influencing Factors Considered Comprehensively: A Case Study from China
}

\author{
Wei Sun, Cuiping Sun, Zhaoqi Li* \\ Department of Business Administration, North China Electric Power University, Baoding, China
}

Received: 6 October 2019

Accepted: 15 November 2019

\begin{abstract}
With the continuous emission of greenhouse gases, the carbon trading market has become a powerful weapon to contain it. It is indispensable to analyze the carbon price of China that acts as the largest emitter of carbon dioxide worldwide. Therefore, this paper proposes an innovative hybrid carbon price forecasting model that incorporates fast ensemble empirical mode decomposition (FEEMD) and extreme learning machine optimized by particle swarm optimization (PSO-ELM) with external and internal influencing factors considered. The original carbon price series are disassembled into several intrinsic mode functions (IMFs) and one residual via FEEMD. The PSO-ELM is then employed to forecast the sub-series. It's remarkable that the inputs of the PSO-ELM model are divided into external and internal influencing factors. Factor analysis is used to extract potential factors from energy prices, macroeconomics and other influencing factors associated with the original carbon price as external influencing factors, and the partial autocorrelation function (PACF) is exploited to select internal influencing factors. A case study in Hubei Province, China shows that the proposed carbon price forecasting model is superior to the contrast models in terms of the smallest prediction error $(\mathrm{MAE}=0.1274$ yuan, MAPE $=0.8368 \%)$ and the strongest stability $(\mathrm{RMSE}=0.0116$ yuan $)$. And the forecasting results demonstrate that the developed model with external and internal influencing factors considered can highly improve carbon price prediction performance and have potential in a wider range of carbon price forecasting. In addition, accurate carbon price forecasting can help the government realize macro control and the investors fulfill risk minimization.
\end{abstract}

Keywords: carbon price forecasting, external and internal influencing factors, fast ensemble empirical mode decomposition, extreme learning machine, particle swarm optimization

*e-mail: 1428571545@qq.com 


\section{Introduction}

Excessive greenhouse gas emissions and consequent adverse impacts on global climate change have become a serious threat to human sustainable development, drawing increased attention from the most international society. Among all greenhouse gases, carbon dioxide is the most prominent and artificially controllable. For the target of global greenhouse gas emissions reduction the carbon market, an effective mechanism for harnessing related issues about global climate change, has emerged. China ranks as the largest emitter of carbon dioxide globally, which has distinct potential to achieve emissions-reduction pledges in the decades to come. Driven by the situation of emission reduction at home and abroad, the Chinese National Development and Reform Commission piloted seven carbon emissions trading markets in 2011, providing an important demonstration for the construction of a nationwide carbon emissions trading system, as well as a valuable opportunity for the development of related companies in China. In view of this, it is crucial to accurately predict carbon prices. Grasping the basic and inherent characteristics of carbon price volatility in advance will not only assist in the formation of appropriate policies and regulations for government to macro-market regulation and climate change response, but also help investors take timely steps to reduce investment risks. Therefore, carbon price prediction is now one of the hottest and most attractive core research topics in the field of energy and climate economics.

Previous research on carbon price forecasting has usually been multi-factor forecasting or pure time series forecasting. Multi-factor forecasting predicts carbon price on the basis of the influencing factors of carbon price. For example, Brent oil, coal and electricity prices [1]; economic and energy indicators [2]; crude oil volatility index (OVX) [3]; and coal, temperature and air quality index [4] are commonly extracted as latent predictors that inherently have strong correlation with future carbon prices during the modeling procedure. Whereas time series forecasting only uses the historical data of carbon price, and time-lagged factors can characterize the development trend and law of original data, which has been researched [5-8]. Incorporating external and internal influencing factors into account comprehensively enables us to extract sufficient information as for precise predictions and countermeasures formulation $[9,10]$. However, as far as is known, few relevant papers comprehensively consider external factors and internal factors when predicting carbon price.

In order to further fill the research gap for carbon price forecasting, this paper comprehensively selects more appropriate and reasonable impact indicators from energy price, macroeconomics and other influencing factors as external influencing factors, meanwhile taking the time-lagged sequence of historical carbon price as the internal influencing factors through PACF.
The selected internal and external influencing factors are utilized as inputs to the forecasting model to predict carbon price.

Accurate predictions depend not only on reasonable variables, but also on prediction methods. Methods applied for carbon price forecasting are mainly branched into three representatives recently: statistical and econometric methods, artificial intelligence techniques and hybrid (ensemble) models.

The statistical and econometric methods can deal with the carbon price time series and obtain good prediction results, including nonparametric modeling technique [11], GARCH-type model [12], HAR-RV model [13], dynamic model averaging (DMA) model [14], and ARIMA models [15]. With the advent of artificial intelligence (AI) models, it has been found that this method can deal with the nonlinear problem of carbon price changes that cannot be solved by statistical and econometric models. The typical AI models covering the LSSVM method [16], muti-layer perceptron (MLP) neural network [17], neuro-fuzzy controller [18], and ELM [5, 19] have been applied to forecast carbon price and achieved relatively good prediction results. However, carbon pricing is a complex, volatile sequence with nonlinear and non-stationary characteristics that cannot be fully captured by a single model.

In order to address the shortcomings, the ensemble (hybrid) models are widely used for carbon price prediction and have achieved good results $[20,21]$. The recent hybrid forecasting model is usually formed by adding signal processing techniques to an AI model, with the aim of further decomposing a nonlinear carbon price time series into more stationary and regular subseries, so that the ultimate forecasting result can be obtained by aggregating the forecast values from the sub-series. Sun et al. demonstrated that the proposed hybrid model based on variational mode decomposition (VMD) and spiking neural networks (SNNs) is appropriate for carbon price forecasting [22]. In order to obtain a more robust method for predicting carbon prices, Zhu proposed a multiscale ensemble forecasting model that includes the empirical mode decomposition (EMD), genetic algorithm and artificial neural network for carbon price forecasting [23]. In addition, Zhu et al. came up with a combination of EMD and evolutionary least squares support vector regression based on particle swarm optimization (PSO), and proved that the proposed model is superior to the other popular forecasting models [24]. Among many decomposition methods, the fast ensemble empirical mode decomposition (FEEMD) is widely adopted to cope with the non-stationary characteristics of original series due to be considered as an optimization method that can overcome the inherent defects of EMD and has strong computing power. The FEEMD method can decompose the original unstable and high volatility time series into several more stationary and regular sub-series in an efficient way to further prediction. So far, it has been applied to related fields, for example wind speed forecasting [25-27], 
pollutant concentration forecasting [28], etc., and has achieved satisfactory prediction results.

Summing up previous research can shed light on the hybrid model combining artificial intelligence models with decomposition methods as a kind of popular model for processing and predicting carbon prices, and the FEEMD is an excellent enough decomposition technique. The extreme learning machine (ELM), a neural network model with superior performance, is distinguished from traditional ones owing to its faster convergence speed, less artificial involvement and can avoid many problems that probably arise in the gradientdriven approach, such as stopping criteria, learning rate and learning epochs. In addition, considering the inherent instability of ELM, the particle swarm optimization (PSO) is exploited to optimize the weight and bias for input layer and hidden layer respectively in ELM. Thus, a hybrid model combining the fast ensemble empirical mode decomposition and the extreme learning machine optimized by particle swarm optimization (FEEMD-PSO-ELM) is first exploited in this paper to predict carbon prices.

The contributions of the present research may be summarized in the following aspects. First, taking Hubei carbon emission trading market as an example, the influencing factors of carbon price are divided into external and internal influencing factors. Regarding the external influence factors, this paper comprehensively considers 19 external influencing factors and selects indicators that are related to carbon prices. In order to decrease the dimensions of variables without distortion, factor analysis is introduced to dig out potential factors hidden in these variables as inputs to the proposed model. Secondly, the latest FEEMD is used to transform the original carbon price into multiple empirical modes. The partial auto correlation function (PACF) is then employed to select the lags for these modes, and the number of lags for these modes obviously that have a strong correlation with the original series will be selected as intrinsic influencing factors. Finally, the extreme learning machine based on particle swarm optimization is applied to predict the carbon price of Hubei Emission Exchange. Through case analysis, compared with contrast models, the proposed model has higher accuracy in predicting carbon price, and the influencing factors added to carbon price forecasting are effective, which further improves the accuracy of the prediction results.

\section{Material and Methods}

This paper applies the carbon price prediction model of PSO-ELM. Firstly, the FEEMD is used to decompose the original time series characterized by high volatility and instability into several subseries; subsequently, input selection is executed where bivariate correlation analysis and factor analysis are applied to screen the main external influencing factors, and PACF is employed to extract internal influencing factors; then the PSO is applied to optimize the ELM model, and the parameters are trained using historical data to obtain the optimal penalty and nuclear parameters to obtain the final prediction model.

\section{Fast Ensemble Empirical Mode Decomposition} (FEEMD)

The ensemble empirical mode decomposition (FEEMD) is developed based on the empirical mode decomposition (EMD) method [29] and ensemble empirical mode decomposition (EEMD) [30]. It adds Gaussian white noise with amplitude $\varepsilon$ to original series, and uses ensemble averaging to eliminate the modal aliasing phenomenon [31]. At the meantime, it can solve the issue of computational complexity and long calculation time of EMD and EEMD.

Two major parameters set in FEEMD are $p$ and $M$ which represent the amplitude of the white noise and the replicated times of EMD respectively. According to the characteristics of the data in this paper, amplitude $p$ is $0.05-0.5$ times, and replicated times $M$ is 100 . Related procedures of FEEMD are described below:

1. Add the random Gaussian white noise series $n_{l}(t)$ to original time series $x(t)$

$$
x_{l}(t)=x(t)+n_{l}(t)
$$

...where $x_{l}(t)$ denotes the noise-added signal utilized in FEEMD of the $l$ th trial.

2. Decompose the noise-added signal $x_{l}(t)$ into several IMFs $c_{i, l}(t), i=1,2, \ldots, n$ and $a$ residue $r_{n, l}(t)$ by adopting the EMD approach.

3. Change different white noise series and repeat procedure (1) to procedure (2) until $l=M$.

4. Calculate the ensemble mean $c_{i}(t)$ of every IMF and residue $r_{n}(t)$ using equations as follows:

$$
\begin{aligned}
& c_{i}(t)=\sum_{l=1}^{M} c_{i, l}(t) / M \\
& r_{n}(t)=\sum_{l=1}^{M} r_{n, l}(t) / M
\end{aligned}
$$

\section{Particle Swarm Optimization Algorithm}

Particle swarm optimization (PSO) was inspired based on studies of predation behavior of birds. This algorithm abstracts the birds in the group into "particles", which are randomly distributed, and then sorts the particles according to the relevant information about the position of each particle in the space, and simultaneously records the optimal historical information of each particle motion until the entire particles find the optimal solution to the problem or meet other stopping conditions [32]. 
Suppose that in the $D$-dimensional search space, $N$ particles are included. In order to find the optimal position, a particle's position calculated by fitness function was updated by tracking two extremes: the optimal location of the particle itself $p_{i}=\left(p_{i 1}, p_{i 2}, \ldots, p_{i D}\right)$ and the global best position of the whole swarm currently $p_{g}=\left(p_{g 1}, p_{g 2}, \ldots, p_{g D}\right)$. Particles in space have two characteristics of position and velocity. In each iteration, velocity $v_{i j}$ and position $x_{i j}$ of the particles are updated according to the following expression:

$$
\begin{gathered}
v_{i j}{ }^{k+1}=\omega v_{i j}{ }^{k}+c_{1} \operatorname{rand}_{1}\left(p_{i j}{ }^{k}-x_{i j}{ }^{k}\right)+c_{2} \operatorname{rand}_{2}\left(p_{g D}{ }^{k}-x_{i j}{ }^{k}\right) \\
x_{i j}{ }^{k+1}=x_{i j}{ }^{k}+v_{i j}{ }^{k+1}
\end{gathered}
$$

...where $k$ is the current number of iterations, $i=1,2, \ldots$ $N, j=1,2, \ldots D, v_{i j} \in\left[-v_{\max }, v_{\max }\right]$, and $v_{\max }$ is a constant. $x_{i j} \in\left[-X_{\max }, X_{\max }\right] X_{\max }$ is also a constant. Both rand $_{1}$ and rand $_{2}$ represent random numbers among 0 to $1 . c_{1}$ and $c_{2}$ are acceleration factors equal to $1.49445 . \omega$ is the inertia weight and its value is non-negative, having great effects on the convergence speed and accuracy of the PSO algorithm, and a larger value of $\omega$ means the algorithm performance better in global optimization and worse in local optimization; and vice versa. The following is the expression of $\omega$ :

$$
\omega=\left(\omega_{s}-\omega_{e}\right)\left(t_{m}-t\right) / t_{m}+\omega_{e}
$$

...where $\omega_{S}$ and $\omega_{e}$ represent the initial inertia weight and the end weight, respectively. $t_{m}$ is the maximum allowed number of iterations.

\section{Extreme Learning Machine (ELM)}

ELM acts as an effective single-hidden layer feedforward neural network (SLFN) and is introduced by Huang et al. [33]. The algorithm randomly initializes the weights and thresholds, and all these are no adjustment required during the whole training process. Once the number of neurons in the hidden layer is set, the only solution with the smallest prediction error can be obtained.

The following is the specific working principle of the ELM network:

1. Define the number $L$ of neurons in the hidden layer, stochastically set the connection weight $\omega$ and the threshold $b$ of the hidden layer neurons as:

$$
\left(\omega_{i}, b_{i}\right), \quad i=1,2, \cdots, L
$$

2. The network output of the activation function $g(x)$ is expressed as:

$$
f_{L}(x)=\sum_{i=1}^{L} \beta_{i} g\left(\omega_{i} * x_{j}+b_{i}\right), x_{j} \in R^{n}, \beta_{i} \in R^{m}
$$

...where the specific form of the hidden layer's output matrix $H$ is shown as:

$$
\begin{gathered}
H\left(\omega_{1}, \cdots, \omega_{L}, b_{1}, \cdots, b_{L}, x_{1}, \cdots x_{N}\right) \\
=\left[\begin{array}{ccc}
g\left(\omega_{1} * x_{1}+b_{1}\right) & \cdots & g\left(\omega_{L} * x_{1}+b_{L}\right) \\
\vdots & \vdots & \vdots \\
g\left(\omega_{1} * x_{N}+b_{1}\right) & \cdots & g\left(\omega_{L} * x_{N}+b_{L}\right)
\end{array}\right]_{N \times L}(9)
\end{gathered}
$$

Thus formula (8) can be simplified as:

$$
\begin{gathered}
H \beta=Y \\
\text { among it } \beta=\left[\begin{array}{c}
\beta_{1}{ }^{T} \\
\beta_{2}{ }^{T} \\
\vdots \\
\beta_{L}{ }^{T}
\end{array}\right]_{L \times m} \text { and } Y=\left[\begin{array}{c}
y_{1}{ }^{T} \\
y_{2}{ }^{T} \\
\vdots \\
y_{N}{ }^{T}
\end{array}\right]_{N \times m}(10)
\end{gathered}
$$

The weight matrix $\beta$ belonging to hidden and output layer can be obtained by the following expression:

$$
\beta=H^{+} Y
$$

...where $\mathrm{H}^{+}$is the Moore-Penrose generalized inverse matrix of hidden layer output matrix $H$.

\section{Approaches of FEEMD-PSO-ELM Model}

As shown in Fig. 1, this framework of the developed carbon price forecasting model consists of three parts, each of which is represented by a different color.

In the green section, the original carbon price series are disassembled into several intrinsic mode functions (IMFs) and one residual via FEEMD. The decomposed IMF components embody local characteristic signals of the original signal at different time scales, which is very helpful for subsequent data processing. In the meantime, the yellow portion of the framework is used to pre-select the input of the PSO-ELM model, which is divided into two parts: external influencing factors and internal influencing factors of the carbon price. The external influencing factors are first screened by bivariate correlation analysis, and then factor analysis is employed to mine potential common factors for the purpose to achieve complexity-reduction of the data by replacing the majority of the original variables with a few variables. The internal influencing factors are selected using partial autocorrelation analysis. Finally, the blue area aims at realizing carbon price prediction by PSO-ELM adopted, and PSO is employed to optimize connection weights $\omega$ and bias threshold $b$ of ELM.

\section{Data Source}

In order to verify the efficiency and practicability of the developed model, this paper analyzes the carbon price and its influence factors of Hubei Emission Exchange in China as the carbon trading volume 


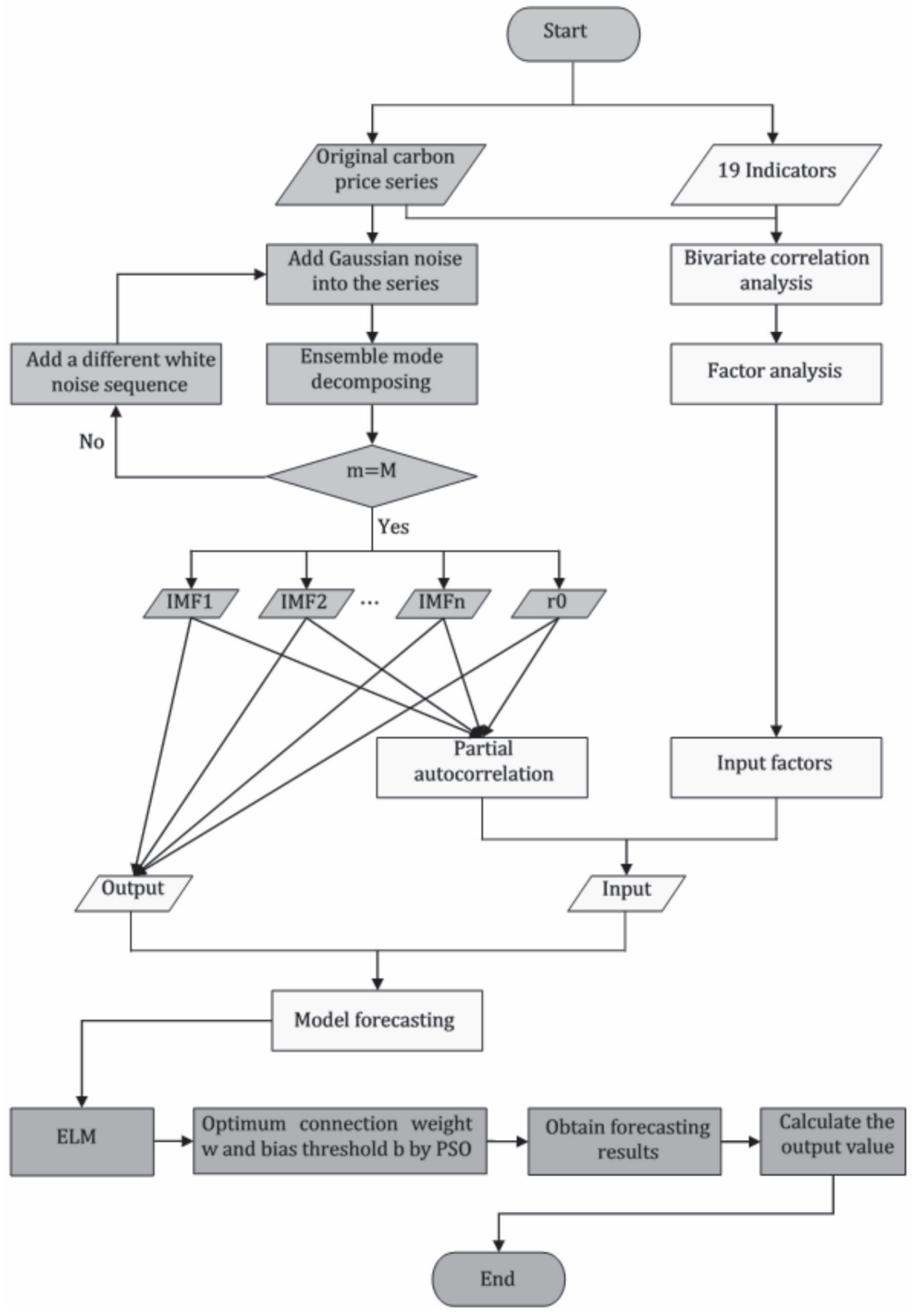

Fig. 1. Flowchart of the proposed carbon price forecasting model FEEMD-PSO-ELM.

of Hubei Emission Exchange is relatively large and persistent. The study period goes from September 1, 2016 to May 4, 2018. The data sample consists of 404 daily observations. Among these, 344 data are employed as a training set while the remaining 60 data are used as a testing set.

\section{Carbon Price Decomposition}

The volatility is violent and the regular information is not clearly available. Thus, FEEMD is utilized to reduce the nonlinearity and high volatility of original carbon price series. The result is distinctly displayed in Fig. 2, on which the raw data is broken down into 6 IMFs and one residual.

For highlighting the superiority of FEEMD, ensemble empirical decomposition (EMD) is also used to disassemble the original series. The EMD also break down the carbon price series into 6 IMFs and one residual.

\section{Selection of Intrinsic Influencing Factors}

According to characteristics of time series data, relevant historical data extracted as time-lagged series can characterize the development trend and law of original data, which can improve prediction accuracy. Taking the effect that historical data variables put on the prediction results into account, the partial autocorrelogram of each acquired IMF can be obtained by using the partial autocorrelation function (PACF). Fig. 3 shows the PACF results of carbon price decomposition after FEEMD. The given $x_{i}$ is 


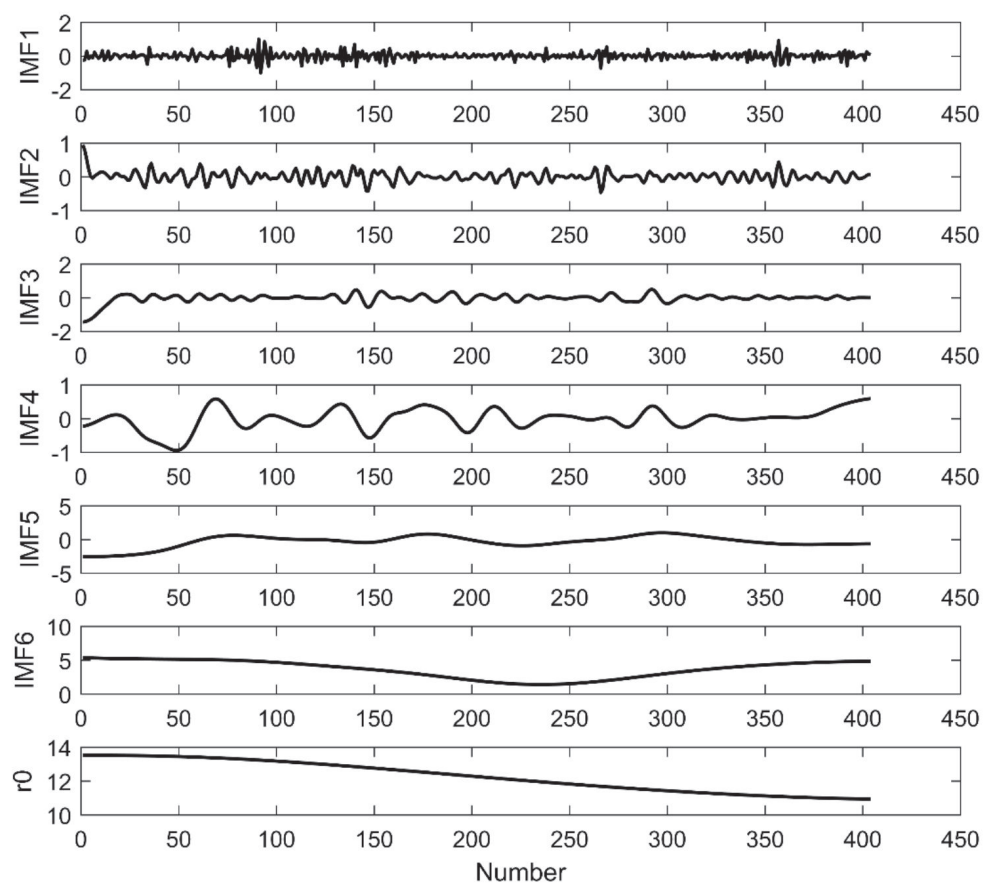

Fig. 2. Decomposed results of Hubei carbon price by FEEMD.

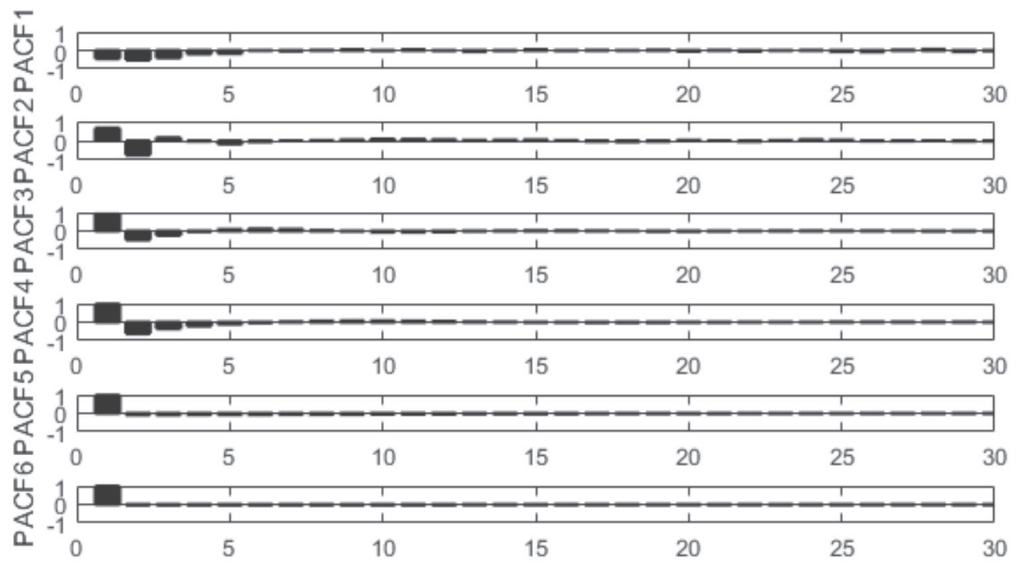

Fig. 3. PCAF results of Hubei carbon price after FEEMD.

the output, and $x_{i-k}$ will be denoted as input variables in such a situation that the PACF at $k$ th lag is out of the $90 \%$ confidence interval. In light of the carbon price decomposition PACF results after FEEMD, Table 1 shows the number of lags for these series obviously that have a strong correlation with the original series.

\section{External Influencing Factors Selection}

According to the previous literature on the influencing factors of carbon prices, it is widely accepted that carbon prices are mainly affected by macroeconomics, energy prices, policy factors, climate and so on. With regard to the external influencing factors affecting carbon prices of the Hubei Emission Exchange in China, combining with the actual situation of the market and the availability of data and taking comprehensive consideration of international carbon prices, macroeconomics, energy prices and other related aspects, this article has pre-selected 19 representative and relatively reasonable influencing indicators: EUA futures price (DEC18), CER futures price (DEC18), SSE (Shanghai Stock Exchange) Composite Index, Shenzhen Component Index, Shanghai Industrial Index, CSI 300 Index, CSI Industrial Index, S \& P 500 Index, British FTSE 100 Index, Zhengzhou Commodity Exchange thermal coal futures price, Shanghai Futures Exchange fuel oil futures price, Dalian Commodity Exchange coking coal futures price, Dalian Commodity Exchange coke futures price, Brent crude oil futures price, Rotterdam coal futures price (DEC18), British natural gas futures price (DEC18), Newcastle coal futures price 
Table 1. Bivariate correlation of carbon price and various factors.

\begin{tabular}{|c|c|c|c|c|c|}
\hline Indicator & Correlation & Significant & Indicator & Correlation & Significant \\
\hline CER futures price(DEC18) & $-0.226 * *$ & 0.000 & CSI 300 Index & $-0.546 * *$ & 0.000 \\
\hline EUA futures price(DEC18) & $0.539 * *$ & 0.000 & CSI Industrial Index & -0.071 & 0.154 \\
\hline S \& P 500 Index & $-0.461 * *$ & 0.000 & Shanghai Industrial Index & $-0.328 * *$ & 0.000 \\
\hline British FTSE 100 Index & $-0.388 * *$ & 0.000 & Shenzhen Component Index & $-0.389 * *$ & 0.000 \\
\hline Euro to RMB exchange rate & $0.491 * *$ & 0.000 & $\begin{array}{l}\text { SSE (Shanghai Stock Exchange) } \\
\text { Composite Index }\end{array}$ & $-0.457 * *$ & 0.000 \\
\hline USD to RMB exchange rate & $-0.804 * *$ & 0.000 & $\begin{array}{l}\text { Shanghai Futures Exchange fuel oil } \\
\text { futures price }\end{array}$ & 0.086 & 0.084 \\
\hline Brent crude oil futures price & -0.081 & 0.106 & $\begin{array}{l}\text { Dalian Commodity Exchange coke } \\
\text { futures price }\end{array}$ & $-0.455^{* *}$ & 0.000 \\
\hline $\begin{array}{l}\text { Newcastle coal futures } \\
\text { price(DEC19) }\end{array}$ & $-0.329 * *$ & 0.000 & $\begin{array}{l}\text { Dalian Commodity Exchange coking } \\
\text { coal futures price }\end{array}$ & -0.090 & 0.070 \\
\hline $\begin{array}{l}\text { Rotterdam coal futures } \\
\text { price(DEC18) }\end{array}$ & $-0.484 * *$ & 0.000 & $\begin{array}{l}\text { Zhengzhou Commodity Exchange } \\
\text { thermal coal futures price }\end{array}$ & $-0.480 * *$ & 0.000 \\
\hline $\begin{array}{l}\text { British natural gas futures } \\
\text { price(DEC18) }\end{array}$ & $-0.206 * *$ & 0.000 & & & \\
\hline
\end{tabular}

Note: ** indicates a significant correlation at the bilateral significance level of 0.01 .

(DEC19), USD to RMB exchange rate, and the Euro to RMB exchange rate.

Exploring the relationship between carbon price and the 19 pre-selected external influencing factors is critical to building a good predictive model. To this end, in this paper the bivariate correlation analysis and the two-tailed significance test are utilized to test the correlation between carbon price and preliminary chosen influencing factors, and Pearson coefficient reflects the correlation. The values of the correlation coefficients are listed in Table 2. It can be found that the 15 concomitant probability values for the two-tailed significance test are 0.000 less than 0.01 , which reveals that there is a significant correlation between carbon price and the 15 aforementioned indicators. However, there is no significant correlation between the other indicators and the carbon price respectively, so these four indicators - CSI Industrial Index, Brent crude oil futures price, Shanghai Futures Exchange fuel oil futures price, and Dalian Commodity Exchange coking coal futures price - will be eliminated.

Although the choice of influencing factors is feasible, intrinsic correlation among these factors may

Table 2. KMO and Bartlett test.

\begin{tabular}{|c|c|c|}
\hline \multicolumn{2}{|c|}{ Index } & Value \\
\hline \multicolumn{2}{|c|}{ KMO } & 0.795 \\
\hline \multirow{3}{*}{$\begin{array}{c}\text { Bartlett } \\
\text { test }\end{array}$} & Approximate Chi-square & 11537.582 \\
\cline { 2 - 3 } & Degree of freedom & 105 \\
\cline { 2 - 3 } & Prob. & 0.000 \\
\hline
\end{tabular}

bring some inconvenience and bias to subsequent research to a certain extent. Hence, factor analysis in SPSS is exploited to address the above problems by further identifying potential common factors that actually affect the subject of the experiment.

The factor analysis results are shown in Tables 3 and 4. The Kaiser Meyer Olkin (KMO) and Bartlett's tests are performed prior to factor analysis. The KMO test is traditionally employed to check the correlation and partial correlation between variables, ranging from 0 to 1 , while Bartlett's test is employed to examine the correlation between variables in the correlation matrix, and whether the correlation matrix is a unit matrix or not. As illustrated in Table 3, the statistical value of KMO is 0.795 (very close to 0.8 ), indicating that factor analysis could be conducted in this paper. At the same time, the value of Bartlett's test is 0.000 , which means the null hypothesis is rejected, that is, negating the idea that the correlation matrix is a unit matrix. Table 4 includes three common factors hidden in the 15 indicators whose cumulative contribution rate up to $86.094 \%$ is indicated as F1, F2 and F3. In order to better explain the extracted common factors, factor rotation is also performed. Meanwhile, based on the correlation and economic connotation of the indicators, F1, F2 and F3 are separately defined as international energy price factor, domestic comprehensive factor and international economic factor. Three specific factor variables can be obtained by the conversion of the scoring coefficients in Table 4. Hence, the following study will take these three factors as external input factors to predict carbon pricing of the Hubei Emission Exchange. 
Table 3. Factor analysis of the remaining 15 variables.

\begin{tabular}{|c|c|c|c|c|c|c|}
\hline Indicator & $\begin{array}{l}\text { Loading } \\
\text { of F1 }\end{array}$ & $\begin{array}{l}\text { Loading } \\
\text { of F2 }\end{array}$ & $\begin{array}{l}\text { Loading } \\
\text { of F3 }\end{array}$ & $\begin{array}{l}\text { Scoring coefficient } \\
\text { of F1 }\end{array}$ & $\begin{array}{l}\text { Scoring coefficient } \\
\text { of F2 }\end{array}$ & $\begin{array}{l}\text { Scoring coefficient } \\
\text { of F3 }\end{array}$ \\
\hline S \& P 500 Index & 0.638 & 0.314 & 0.662 & 0.073 & -0.090 & 0.202 \\
\hline British FTSE 100 Index & 0.045 & 0.162 & 0.924 & -0.190 & -0.128 & 0.508 \\
\hline CER futures price(DEC18) & -0.422 & -0.314 & -0.796 & 0.038 & 0.088 & -0.324 \\
\hline USD to RMB exchange rate & 0.504 & 0.279 & 0.607 & -0.288 & 0.061 & 0.130 \\
\hline Euro to RMB exchange rate & -0.162 & -0.225 & -0.901 & 0.036 & -0.075 & 0.203 \\
\hline EUA futures price(DEC18) & 0.959 & 0.106 & 0.084 & 0.342 & -0.103 & -0.164 \\
\hline $\begin{array}{l}\text { Newcastle coal futures } \\
\text { price(DEC19) }\end{array}$ & 0.736 & 0.474 & 0.352 & 0.151 & 0.028 & -0.036 \\
\hline $\begin{array}{l}\text { Rotterdam coal futures } \\
\text { price(DEC18) }\end{array}$ & 0.707 & 0.430 & 0.525 & 0.111 & -0.022 & 0.082 \\
\hline $\begin{array}{l}\text { British natural gas futures } \\
\text { price(DEC18) }\end{array}$ & 0.844 & 0.272 & 0.238 & 0.244 & -0.049 & -0.083 \\
\hline CSI 300 Index & 0.580 & 0.580 & 0.527 & 0.041 & 0.059 & 0.078 \\
\hline Shanghai Industrial Index & 0.125 & 0.881 & 0.317 & -0.135 & 0.291 & -0.018 \\
\hline Shenzhen Component Index & 0.310 & 0.852 & -0.017 & 0.008 & 0.323 & -0.247 \\
\hline $\begin{array}{c}\text { SSE (Shanghai Stock } \\
\text { Exchange) Composite Index }\end{array}$ & 0.093 & 0.857 & 0.453 & -0.172 & 0.258 & 0.075 \\
\hline $\begin{array}{c}\text { Dalian Commodity } \\
\text { Exchange coke futures price }\end{array}$ & 0.303 & 0.655 & 0.232 & -0.018 & 0.189 & -0.057 \\
\hline $\begin{array}{l}\text { Zhengzhou Commodity } \\
\text { Exchange thermal coal futures } \\
\text { price }\end{array}$ & 0.280 & 0.814 & 0.172 & -0.036 & 0.272 & -0.121 \\
\hline Cumulative contribution rate & $33.023 \%$ & $63.092 \%$ & $86.094 \%$ & & & \\
\hline
\end{tabular}

\section{Comparative Framework}

As shown in Fig. 4, seven different models are employed to contrast with the established model, which can be divided into two parts. In part 1, three models including FEEMD-PSO-ELM, EMD-PSO-ELM and PSO-ELM are introduced to illustrate the essential of introducing FEEMD. The applicability and effectiveness of PSO-ELM in carbon price forecasting can be

Table 4. PACF results after FEEMD.

\begin{tabular}{|c|c|}
\hline & Carbon price \\
\hline IMFs and $r_{0}$ & Lag \\
\hline IMF1 & $\left(x_{t-1}, x_{t-2}, x_{t-3}, x_{t-4}, x_{t-5}\right)$ \\
\hline IMF2 & $\left(x_{t-1}, x_{t-2}, x_{t-3}, x_{t-5}\right)$ \\
\hline IMF3 & $\left(x_{t-1}, x_{t-2}, x_{t-3}, x_{t-6}\right)$ \\
\hline IMF4 & $\left(x_{t-1}, x_{t-2}, x_{t-3}, x_{t-4}, x_{t-5}\right)$ \\
\hline IMF5 & $\left(x_{t-1}\right)$ \\
\hline IMF6 & $\left(x_{t-1}\right)$ \\
\hline$r_{0}$ & $\left(x_{t-1}\right)$ \\
\hline
\end{tabular}

verified in Part 2. Moreover, one find is at the heart of taking these two parts into comparison: adoption of decomposition is conducive to largely improve the accuracy of the prediction model. The inputs for all of the above models are internal and external influencing factors selected in this paper.

\section{Model Performance Evaluation}

To ascertain which forecasting model is more superior, MAPE, MAE, and RMSE, denoting mean absolute percentage error, mean absolute error and root mean square error respectively are selected to measure performances of all models involved. The smaller the values, the better the forecasting performance. The formulas of these three indexes are defined as follows:

$$
\begin{gathered}
M A P E=\frac{1}{n} \sum_{i=1}^{n}\left|\frac{y_{i}-\hat{y}_{i}}{y_{i}}\right| * 100 \% \\
M A E=\frac{1}{n} \sum_{i=1}^{n}\left|y_{i}-\hat{y}_{i}\right|
\end{gathered}
$$




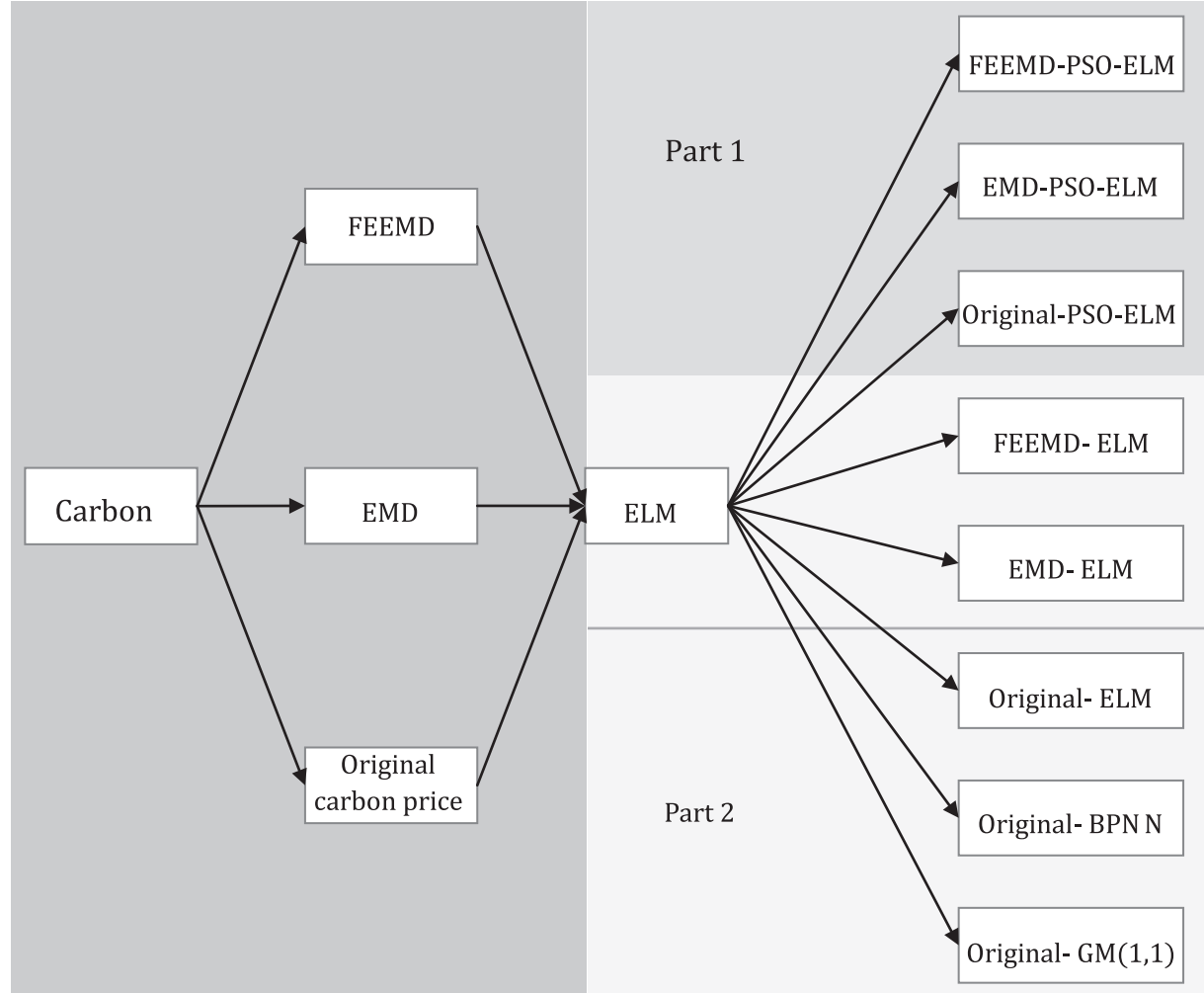

Fig. 4. Framework of comparison models.

$$
R M S E=\sqrt{\frac{1}{n} \sum_{i=1}^{n}\left|\frac{y_{i}-\hat{y}_{i}}{y_{i}}\right|^{2}}
$$

\section{Results and Discussion}

The procedures of FEEMD and PSO-ELM were executed by MATLAB R2016a. Fig. 5 demonstrates the carbon price prediction fitting curves for 60 data from January 30 to May 4, 2018 obtained by eight different models. It can be seen that: (a) in comparison with other seven models, the FEEMD-PSO-ELM model has the highest goodness-of-fit between the predicted and actual values, while the $\operatorname{GM}(1,1)$ results are the worst; (b) the fitting effect of ELM-based models is superior to other models in general; and (c) compared with the model without the decomposition algorithm, the model incorporating the decomposition algorithm has higher prediction accuracy.

Table 5 clearly listed the statistical errors for quantitatively evaluating the six prediction models. In order to display the comparison results more clearly and intuitively, a histogram is shown in Fig. 6.

Combining Table 5 and Fig. 6, the following important conclusions can be drawn:

1. Among the four prediction models of ELM, BPNN, and $\operatorname{GM}(1,1)$, the MAPE, MAE and RMSE of ELM are all the smallest, then BPNN, and the largest is $\operatorname{GM}(1,1)$, which suggests that the selected model ELM is tremendously effective for predicting carbon price as the strong global search capability.

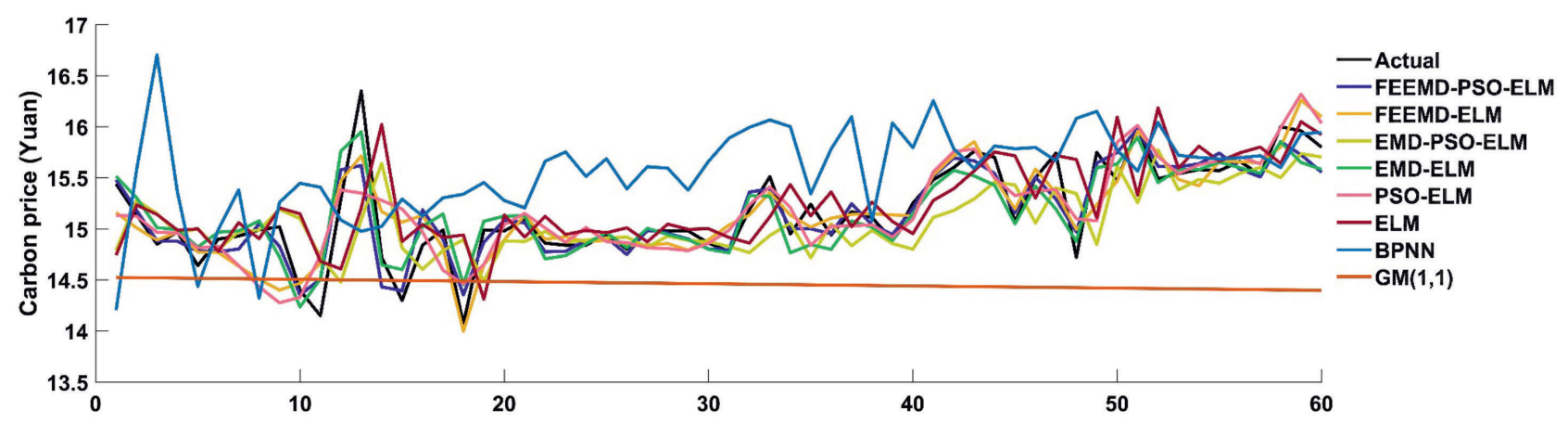

Fig. 5. Carbon price forecasting results between the proposed models and other contrast models. 
Table 5. Three evaluation indicators of models in carbon price forecasting.

\begin{tabular}{|c|c|c|c|c|c|c|c|c|}
\hline Index & $\begin{array}{c}\text { FEEMD-PSO- } \\
\text { ELM }\end{array}$ & $\begin{array}{c}\text { FEEMD- } \\
\text { ELM }\end{array}$ & $\begin{array}{c}\text { EMD-PSO- } \\
\text { ELM }\end{array}$ & EMD-ELM & PSO-ELM & ELM & BPNN & GM(1,1) \\
\hline MAPE (\%) & 0.8368 & 0.9287 & 1.2455 & 1.4509 & 2.0544 & 2.1220 & 3.7024 & 4.82287 \\
\hline MAE (RMB Yuan) & 0.1274 & 0.1409 & 0.1886 & 0.2193 & 0.3124 & 0.3207 & 0.5554 & 0.74287 \\
\hline RMSE (RMB Yuan) & 0.0116 & 0.0123 & 0.0168 & 0.0205 & 0.0273 & 0.0288 & 0.0465 & 0.05486 \\
\hline
\end{tabular}
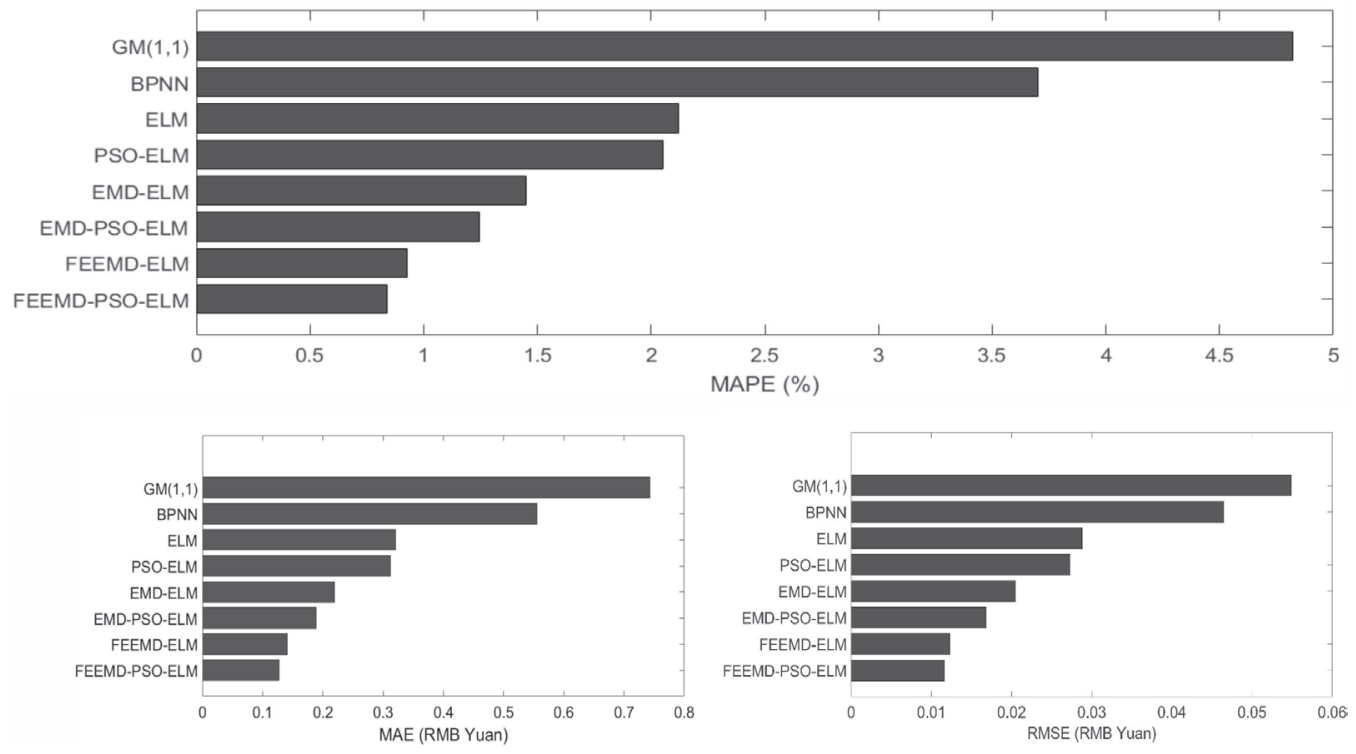

Fig. 6. Three evaluation indicators of models in carbon price forecasting.

2. On the basis of the order of MAPE, MAE and RMSE, the PSO-ELM and ELM index values are 2.0544\%, 0.3124 yuan, 0.0273 yuan and $2.1220 \%, 0.3207$ yuan, and 0.0288 yuan, respectively, indicating that ELM optimized by PSO can improve prediction accuracy and reinforce sensitivity. The FEEMD-PSO-ELM and FEEMD-ELM also showed the same conclusion. These are all due to the PSO's strong global search capability overcoming the inherent instability of ELM.

3. The three models from the best to the worst are FEEMD-PSO-ELM, EMD-PSO-ELM and PSOELM, which certifies that regardless of the type of the decomposition method, there is a positive effect of improving forecasting precision. In addition, the performance of FEEMD-ELM, EMD-ELM, and ELM also reached the same conclusion. As can be seen from Table 5, there is a decrease between the EMD-driven model and the FEEMD-driven model in terms of predictive performance. For example, the MAPE of FEEMD-PSO-ELM is $0.8368 \%$ while the corresponding value of EMD-PSO-ELM is $1.2455 \%$. The reason for this phenomenon is, to a great extent, thanks to the uniform frequency distribution in Gaussian white noise of FEEMD, which can solve inherent pitfalls, pattern mixing problem, and exiting in EMD. Overall, it can be concluded that the FEEMD-PSO-ELM is the best in line with these three statistic assessment indicators.

For all the above analysis results, the input values of these models are the three exogenous factors, which indicates that not only the extracted factors but also the FEEMD-PSO-ELM model have been proved to be available and effective.

Furthermore, only the lag period of the original carbon price data is used as input for the FEEMDPSO-ELM model. The results are clearly displayed in Table 6. For this developed model, the three metrics values MAPE, MAE and RMSE of the prediction result about the input with external influencing factors are all less than the metrics values of the prediction result that the input without external influencing factors. It is further verified that forecasting results is significantly

Table 6. Three evaluation indicators for different inputs of the FEEMD-PSO-ELM model.

\begin{tabular}{|c|c|c|}
\hline Index & $\begin{array}{c}\text { Input with } \\
\text { influencing factors }\end{array}$ & $\begin{array}{c}\text { Input without } \\
\text { influencing factors }\end{array}$ \\
\hline MAPE (\%) & 0.8368 & 1.1308 \\
\hline MAE (RMB Yuan) & 0.1274 & 0.1706 \\
\hline RMSE (RMB Yuan) & 0.0116 & 0.0149 \\
\hline
\end{tabular}


pretty with external influencing factors added into lag period of the original carbon price. The reasons for this result may be: (a) the industrial structure of Hubei Province in China is mainly industrial, and with obvious characteristics of heavy chemical industry, and the demand for energy is large. The participants in the carbon trading market are mainly industrial enterprises with large energy consumption, so Hubei's carbon prices are greatly affected by energy prices [34]. (b) Due to the superior geographical environment of Hubei Province, economic development has been good and fast in recent years, attracting more domestic and foreign enterprises to invest here, so that its development is closely related to the economic situation at home and abroad. However, economic development is bound to lead to an increase in carbon dioxide emissions, inevitably increasing the demand for carbon allowances and further affecting the price of the carbon market [35].

\section{Conclusions}

A novel ensemble model is proposed in this paper involving fast ensemble empirical mode decomposition, particle swarm optimization and extreme learning machine, and with external and internal influencing factors considered comprehensively, aiming at carbon price precisely forecasting Owing to the volatility and nonlinearity of carbon price series, FEEMD is employed to filter the noise and then PSO-ELM is used to predict the decomposed series. For intrinsic influencing factors, PACF is utilized to select time-lagged variables of the carbon price series as inputs. In addition, 19 indexes from energy price, macroeconomics and other influencing factors related to the original carbon price are preliminary chosen as external influencing factors, then 3 factors are ultimately identified through factor analysis as the inputs of the proposed model for purposes of reducing information overlapp and improving prediction accuracy. To verify practicability and effectiveness, a case study was conducted on the carbon price of the Hubei Emission Exchange and empirical results show satisfactory accuracy of the proposed model that take external and internal influencing factors into account - especially energy prices and macroeconomics derived from external influencing factors.

Comparison between the developed model and other models make it clear that: (a) from factor analysis of 19 preliminarily selected external influencing indicators to mine latent 3 variables, as well extract internal influencing factors by PACF rendering it theoretically and practically available to boost predicting accuracy. (b) Integrating FEEMD with PSO-ELM is a way to blaze a new trail for predicting carbon price series with high volatility and nonlinearity. (c) The PSO-ELM model, distinct from other techniques, possesses the best prediction accuracy and fastest calculation speed due to the entry of particle swarm optimization algorithm which enhances the global optimization ability. (d) The case study of the carbon price of Hubei Emission Exchange proved that the proposed model with external and internal influencing factors considered comprehensively, especially energy prices and macroeconomics, are effective and promising.

Based on our results, the predicting results are of great significance in the following three aspects: (1) guiding investors to choose a reasonable investment plan to minimize risks; (2) inspire policymakers to institute reasonable policies and regulation aimed at mitigating climate change and promoting China's macroeconomic growth; and (3) further promoting researchers to explore the projected reduction-emission potential of carbon dioxide.

\section{Conflict of Interest}

All authors of this paper declare no conflict of interest.

\section{References}

1. BYUN S.J., CHO H. Forecasting carbon futures volatility using GARCH models with energy volatilities. Energy Econ. 40, 207, 2013.

2. ZHAO X., HAN M., DING L.L., KANG W.L. Usefulness of economic and energy data at different frequencies for carbon price forecasting in the EU ETS. Appl. Energy 216, 132, 2018.

3. DUTTA A. Modeling and Forecasting the Volatility of Carbon Emission Market: The Role of Outliers, Timevarying Jumps and Oil Price Risk. Journal of Cleaner Production 172, 2773, 2018.

4. HAN M., DING L.L., ZHAO X., KANG W.L. Forecasting carbon prices in the Shenzhen market, China: The role of mixed-frequency factors. Energy, 171, 69, 2019.

5. SUN W., DUAN M. Analysis and Forecasting of the Carbon Price in China's Regional Carbon Markets Based on Fast Ensemble Empirical Mode Decomposition, Phase Space Reconstruction, and an Improved Extreme Learning Machine. Energies, 12.2, 277, 2019.

6. ZHU J.M., WU P., CHEN H., LIU J., ZHOU L. Carbon price forecasting with variational mode decomposition and optimal combined model. Physica A: Statistical Mechanics and its Applications, 519, 140, 2019.

7. ZHOU J.G., YU X.C., YUAN X.L. Predicting the carbon price sequence in the Shenzhen emissions exchange using a multiscale ensemble forecasting model based on ensemble empirical mode decomposition. Energies, 11.7, 1907, 2018.

8. CHEN Z., YANG X. Management SO. Forecasting of China's regional carbon market price based on multifrequency combined model. Syst. Eng.-Theory Pract, 36, 3017, 2016.

9. CHEVALLIER J. Volatility forecasting of carbon prices using factor models. Economics Bulletin 30 (6), 1642, 2010.

10. ZHANG L., ZHANG J.B., XIONG T., SU C. Interval forecasting of carbon futures prices using a novel hybrid 
approach with exogenous variables. Discrete Dynamics in Nature and Society. (2017-8-9), 1, 2017.

11. CHEVALLIER J. Nonparametric modeling of carbon prices. Energy Econ. 33 (6), 1267, 2011.

12. BYUN S.J., CHO H. Forecasting carbon futures volatility using GARCH models with energy volatilities. Energy Econ. 40, 207, 2013.

13. CHEVALLIER J., SÉVI B. On the realized volatility of the ECX $\mathrm{CO}_{2}$ emissions 2008 futures contract: distribution, dynamics and forecasting. Ann Finance 7, , 2011.

14. KOOP G., TOLE L. Forecasting the European carbon market. J. R. Stat. Soc. Ser. A 176 (3), 723, 2013.

15. ZHU B.Z., WEI Y.M. Carbon price prediction with a hybrid ARIMA and least squares support vector machines methodology. Omega 41, 517, 2013.

16. ZHU B.Z., WEI Y.M. Carbon price prediction based on integration of GMDH, particle swarm optimization and least squares support vector machines. Syst. Eng.- Theory Pract. 31 (12), 2264, 2011.

17. Fan X.H., Li S.S., TIAN L.X. Chaotic characteristic identification for carbon price and a multi-layer perception network prediction model. Expert Syst Appl. 42, 3945, 2015.

18. ATSALAKIS G.S. Using computational intelligence to forecast carbon prices. Appl. Soft Comput. 43, 107, 2016.

19. SUN W., ZHANG C.C., SUN C.P. Carbon pricing prediction based on wavelet transform and K-ELM optimized by bat optimization algorithm in China ETS: the case of Shanghai and Hubei carbon markets. Carbon Management, 9.6, 605, 2018.

20. SEIFERT J., UHRIG-HOMBURG M., WAGNER M. Dynamic behavior of $\mathrm{CO}_{2}$ spot price. J. Environ. Econ. Manag. 56, 180, 2008.

21. ZHANG Y.J., WEI Y.M. An overview of current research on EU ETS: Evidence from its operating mechanism and economic effect. Appl. Energy 87, 1804, 2010.

22. SUN G.Q., CHEN T., WEI Z.N., SUN Y., ZANG H., CHEN S. A carbon price forecasting model based on variational mode decomposition and spiking neural networks. Energies 9, 54, 2016.

23. ZHU B.Z. A novel multiscale ensemble carbon price prediction model integrating empirical mode decomposition, genetic algorithm and artificial neural network. Energies 5, 355, 2012.

24. ZHU B., HAN D., WANG P., WU Z., ZHANG T., WEI Y.M. Forecasting carbon price using empirical mode decomposition and evolutionary least squares support vector regression. Applied energy, 191, 521, 2017.

25. LIU H., TIAN H., LIANG X., Li Y. New wind speed forecasting approaches using fast ensemble empirical model decomposition, genetic algorithm, Mind Evolutionary Algorithm and Artificial Neural Networks. Renewable Energy, 83, 1066, 2015.

26. LIU H., TIAN H.Q., LIANG X.F., Li Y.F. Wind speed forecasting approach using secondary decomposition algorithm and Elman neural networks. Applied Energy, 157, 183, 2015.

27. SUN W., LIU M.H. Wind speed forecasting using FEEMD echo state networks with RELM in Hebei, China. Energy Conversion and Management, 114, 197, 2016.

28. LUO H., WANG D., YUE C., LIU Y., GAO H. Research and application of a novel hybrid decomposition-ensemble learning paradigm with error correction for daily $\mathrm{PM}_{10}$ forecasting. Atmospheric Research, 201, 34, 2018.

29. HUANG N.E., SHEN Z., LONG S.R. The empirical mode decomposition and the Hilbert spectrum for nonlinear and non-stationary time series analysis. Process Roy Soc London A454, 903, 1998.

30. WU Z., HUANG N.E. Ensemble empirical mode decomposition: a noise-assisted data analysis method. Adv. Adapt Data Anal. 1, 1, 2009.

31. WANG Y.H., YEH C.H., YOUNG H.W.V., HU K., LO M.T. On the computational complexity of the empirical mode decomposition algorithm. Physica A 400, 159, 2014.

32. KENNEDY J., EBERHART R.C. Particle swarm optimization. In: Proceedings of IEEE International Conference on Neutral Networks (ICNN'95), IV, 1942. Perth, Australia, 1995.

33. HUANG G.B., ZHU Q.Y., SIEW C.K. Extreme learning machine: a new learning scheme of feedforward neural networks. In: Proceedings of the international joint conference on neural networks, IEEE, 985, 2004.

34. YANG Q., GUO S., YUAN W.H., SHEN Q., CHEN Y.Q., WANG X.H., HAYAT T. Energy-dominated carbon metabolism: a case study of Hubei province, China. Ecological Informatics, 26, 85, 2015.

35. WANG Y., YANG S., LIU C., LI S. How Would Economic Development Influence Carbon Productivity? A Case from Hubei in China. International journal of environmental research and public health, 15 (8), 1730, 2018. 